\title{
Correspondence
}

\section{Elimination diets in eczema}

Sir,

We read with interest the paper by $T \mathbf{J}$ David on anaphylactic shock during elimination diets for severe atopic eczema. ${ }^{1}$ We recently observed a similar case. This girl had developed severe diarrhoea at 5 weeks of age while being fed an adapted cows' milk formula (Preaptamil, Milupa). During a milk free diet her symptoms subsided, but diarrhoea recurred when a full amount of adapted cows' milk formula was again given. The infant stayed well and lost little weight. Three such challenges were carried out, but after the third the severe diarrhoea persisted even when lactose-free soya protein milk was given. At age 7 weeks the baby was fed a lactalbuminhydrolysate milk formula (Alfaré, Nestlé) and on this regimen she quickly recovered and gained weight.

Although we believed that she suffered from cows' milk protein intolerance, it was thought that the symptoms might also have been due to a postdiarrhoeal lactose intolerance. At the age of 16 weeks, therefore, after nine weeks of receiving Alfaré, reintroduction of cows' milk formula was attempted. The baby was given $150 \mathrm{ml}$ adapted cows' milk formula (Preaptamil, Milupa) at 11 am. The feed was well tolerated and she stayed asymptomatic. The next feeds were Alfaré at $2 \mathrm{pm}$ and $5 \mathrm{pm}$. Suddenly, $91 / 2$ hours after the cows' milk feed, she was found by the nurse nearly dead, grey and cyanotic, with gasping respirations, and vomited milk was seen around the mouth. She was rescuscitated and treated in the intensive care unit without further complications. No milk was found in the trachea and the chest film showed no aspiration pneumonia. Subsequently, however, spastic tetraplegia developed.

At the time we were uncertain about the aetiology of the shock, because we had never seen delayed anaphylactic shock in cows' milk intolerance. Having read the paper by David we feel that the aetiology in our patient might be similar to that of the patients reported.

Close observation might lead to a more frequent recognition of delayed anaphylactic shock and sequelae in patients challenged after long term elimination diets.

U GoriuP University of Graz, AND I MUTZ Children's Hospital, Loeben, Austria

\section{Dr David comments:}

There is a striking resemblance between this important report, case 4 of the original paper, and the patient mentioned in the first paragraph of the discussion of the paper. The intervals between milk exposure and collapse were $91 / 2$ hours, $101 / 2$ hours, and 8 hours respectively. The delay in onset of anaphylaxis adds a new dimension to the difficulties of food challenges. So far the problem seems to apply only to challenges with cows milk, though there is no reason why it should not occur with other foods. The hazards of delayed anaphylactic reactions to foods have not been clearly recorded before, though it is presumably this kind of disaster that formed the basis for the recommendation that babies having cows' milk challenges should be kept under observation on the ward for 9 to 12 hours. ${ }^{2}$ Given the lack of warning of anaphylaxis, and the very small risk, it is difficult to know what should comprise 'close observation'. Mere hospital admission is plainly no guarantee of safety. For cows' milk challenges we try to ensure that either a nurse or the mother is with the child constantly throughout the 12 hours of inpatient observation which we insist upon, but there are often insufficient nurses. Catastrophic cases such as these are rare. Nevertheless I suspect some under reporting, and I would urge colleagues to publish (or pass to me) details of similar cases.

\section{References}

1 David TJ. Anaphylactic shock during elimination dicts for severe atopic eczema. Arch Dis Child 1984;59:983-6.

2 Anonymous. Infantile bloody diarrhoea and cows' milk allergy [Editorial]. Lancet 1984:i:1159-60.

\section{Sir,}

It is not clear what is special about children with either active atopic eczema or a history of atopic eczema, that makes them react in the manner described by David' in his interesting and important paper.

Of the four boys who developed anaphylaxis, two (cases 1 and 3) showed urticaria as part of the picture and one of these (case 1) showed contact urticaria also; but only one of the four (case 2) seems to have had worsening of his atopic eczema with ingestion of the particular anaphylactic allergen. The negative radio-allergosorbent test and low total $\operatorname{IgE}$ values for cases 3 and 4 are interesting but only add to the puzzle: perhaps case 4 did not have atopic eczema at all. Measurement of serum $\operatorname{IgG} 4$ antibodies against dietary allergens may be helpful in some cases of reactions to foods.

Repeated ingestion of a dietary allergen may result in the development of allergy and the appearance of a diffuse erythematous rash, ${ }^{2}$ urticaria, or even anaphylaxis. Urticaria is not atopic eczema and any atopic eczema already present may not necessarily worsen with exposure to the allergen producing urticaria. It is possible, however. that the widespread itching that accompanies urticaria will tend to make existing eczema more itchy and thus more prone to scratching, and may tend to localise atopic 
eczema appearing later to the most irritant areas. It seems reasonable to suggest that after a time, or with repeated exposure, an allergen initially producing an erythema or urticaria in the atopic patient ceases to cause urticaria but sets off itching and scratching manifesting as eczema. ${ }^{3}$ In practice, however, it is unusual to find atopic eczema starting other than per se and this perhaps offers some evidence in favour of immediate hypersensitivity to dietary allergens playing no great part in the pathogenesis of atopic eczema. Atopic eczema can, of course, worsen with an allergen that does not produce urticaria. Prevention or delayed appearance of cows' milk allergy may be helped by encouraging breast feeding in the early postnatal months, particularly in those with a strong atopic family background $^{4}$ and in infants of low birthweight.

There is obvious wisdom in withdrawing suspect, harmful dietary allergens but David ${ }^{1}$ has illustrated the need for reintroduction to be carried out in hospital if possible. Unfortunately, prick tests and radio-allergosorbent tests cannot be relied on to obviate the occasional need for elimination diets.

\section{Julian Verbov Royal Liverpool Children's Hospital, Liverpool $L 77 D G$}

\section{Dr David comments:}

Urticaria and atopic eczema are both disorders which can occur after the ingestion of certain foods. The speed of onset of the adverse reaction is variable. Both disorders may coexist in the same patient, and the features of one may merge into those of the other. As yet, no laboratory investigation has been found to replace listening to parents. Clinicians who feel the need to equate 'allergy' with IgE mediated hypersensitivity become confused when they discover that only a few of the symptoms or signs are explicable on this basis. IgG4 antibodies to foods impress colleagues, and thereby confer a certain pseudoscientific respectability to the whole murky area of food intolerance, but their estimation is not likely to be of any practical value in the management of children with atopic eczema. The idea that allergic disease can be prevented by encouraging breast feeding is fashionable and attractive. The absence of any good supporting scientific evidence ${ }^{6}$ is most tiresome. It is neither possible, practical, nor rational to perform all food challenges in hospital, and to insist on this is tantamount to excluding the use of elimination diets in the treatment of atopic eczema. It would be a great shame if the small risk of severe anaphylaxis were used as yet another excuse by sceptics of food intolerance to shun elimination diets in children with severe, intractable atopic eczema.

Allergy is a minefield. Maybe the occasional explosion is not so surprising.

\footnotetext{
References

1 David TJ. Anaphylactic shock during climination dicts for severe atopic eczema. Arch Dis Child 1984;59:983-6.

2 Sampson HA, Jolic PL. Increased plasma histamine concentra-
}

tions after food challenges in children with atopic dermatitis. $N$ Engl J Med 1984;311:372-6.

3 Sampson HA. Role of immediate food hypersensitivity in the pathogenesis of atopic dermatitis. J Allergy Clin Immunol 1983:71:473-80.

4 Matthew DJ, Taylor B, Norman AP, Turner MW, Soothill JF Prevention of eczema. Lancet 1977;i:321-4.

- Lucas A. McLaughlan P. Coombs RRA. Latent anaphylactic sensitisation of infants of low birth weight to cows' milk protein. Br Med J 1984:289:1254-6.

6 Van Asperen PP, Kemp AS, Mellis CM. Relationship of diet in the development of atopy in infancy. Clin Allergy 1984;8: $525-32$.

\section{Prevalence of glucose-6-phosphate- dehydrogenase deficiency}

Sir,

Glucose-6-phosphate-dehydrogenase (G6PD) deficiency is probably the most common $\mathrm{X}$ linked disorder in the world. It is well known to cause haemolytic crises and neonatal jaundice, and to occur more frequently in persons of Mediterranean, African, and Asian racial stock. Because we had encountered a number of patients with severe neonatal jaundice, we screened all babies (boys and girls) of African or Asian stock born at this hospital over a three month period. Consent was obtained from the mothers, $(0.1 \mathrm{ml}$ blood was taken by heel prick, and G6PD activity was assayed using a dye reduction method.

Altogether $234(32 \%)$ babies were tested. Twenty two were of Asian descent and 212 were of African descent. Eleven of the 118 boys $(9.3 \%)$ were G6PD deficient and four of the 116 girls $(3.4 \%)$ also had the deficiency. The 15 G6PD deficient babies were followed up, and the deficiency confirmed at the age of 2 months. Five of the 15 had moderate neonatal jaundice but none required treatment and all were clinically well at 2 months of age. Of the 15 mothers of these babies, two proved to have a deficiency, but were unaware of any symptoms attributable to the condition.

A technical problem encountered was that if a baby's blood has a high reticulocyte count, the young red cell population may mask a partial (heterozygote) deficiency. Also, because the white cell count is high in neonates a deficiency may be masked; this can be resolved either by delaying the test until the white cell count is normal, or by centrifuging and discarding the buffy layer.

While we have been aware of the problem of G6PD deficiency causing neonatal jaundice we were, perhaps naively, surprised at the incidence in our health district, especially in girls. Because of the high positive yield and the implied prevention of serious complications, we now perform a G6PD screen on any baby, boy or girl, of African, Asian, or, Mediterranean stock who is jaundiced enough to require a serum bilirubin estimation in the newborn period.

K Shusheela, A Grimes, and J W Scopes St Thomas's Hospital, London SEI $7 E H$ 